\title{
ANDRZEJ DERDZIŃSKI
}

\section{Riemannian metrics with harmonic curvature on 2-sphere bundles over compact surfaces}

\author{
Bulletin de la S. M. F., tome 116, no 2 (1988), p. 133-156 \\ <http://www.numdam.org/item?id=BSMF_1988_116_2_133_0>
}

(C) Bulletin de la S. M. F., 1988, tous droits réservés.

L'accès aux archives de la revue «Bulletin de la S. M. F. » (http: //smf.emath.fr/Publications/Bulletin/Presentation.html) implique l'accord avec les conditions générales d'utilisation (http://www.numdam.org/ conditions). Toute utilisation commerciale ou impression systématique est constitutive d'une infraction pénale. Toute copie ou impression de ce fichier doit contenir la présente mention de copyright.

\section{Numdam}


Bull. Soc. math. France,

116,1988 , p. 133-156.

\title{
RIEMANNIAN METRICS WITH HARMONIC CURVATURE ON 2-SPHERE BUNDLES OVER COMPACT SURFACES
}

PAR

\author{
ANDRZEJ DERDZIŃSKI $\left(^{*}\right)$
}

RÉSUMÉ. - On montre que $S^{2} \times S^{2}$ et d'autres fibrés en sphères $S^{2}$ sur des surfaces admettent des familles continues de métriques riemanniennes à courbure harmonique qui ne sont ni d'Einstein, ni conformément plates et ni localement réductibles. Les exemples obtenus sont caractérisés, à une isométrie locale près, par une condition sur la courbure.

Abstract. - We show that $S^{2} \times S^{2}$ and other $S^{2}$ bundles over surfaces admit continuous families of Riemannian metrics with harmonic curvature that are not Einstein, conformally flat or locally reducible. Our examples are characterized, up to a local isometry, by a curvature condition.

\section{Introduction}

A Riemannian manifold is said to have harmonic curvature if the divergence of its curvature tensor $R$ is identically zero $\left(\nabla^{i} R_{i j k \ell}=0\right)$. For compact manifolds, this means that the Riemannian connection is a Yang-Mills potential in the tangent bundle.

The aim of this paper is to prove the existence of compact fourmanifolds with harmonic curvature that are different from the "classical" examples (Einstein, conformally flat, locally reducible), which answers the questions raised by J.-P. Bourguignon in [5, p. 281] and [6, p. 21]. More precisely, we exhibit curves of metrics of this type on $S^{2} \times S^{2}$ (TheOREM 4) and some of its quotient spaces (CoRollary 2), and higherdimensional families of such metrics on $N \times S^{2}$, on $N \times \mathbb{R} P^{2}$ and on the (unique) nontrivial $\mathrm{SO}(3)$ bundle over $N$ with fibre $S^{2}$ or $\mathbb{R} P^{2}, N$

(*) Texte reçu le 24 juillet 1986.

A. Derdziński, Department of Mathematics, The Ohio State University, Columbus, OH 43210, U.S.A.

Research done at M.S.R.I., Berkeley. Supported in part by NSF Grant 81-20790.

BULlETIN DE LA SOCIÉTÉ MATHÉMATIQUE DE FRANCE 0037-9484/1988/133/ $\$ 5.00$

(C) Société mathématique de France 
being any compact orientable surface of genus $p>1$ (THEOREM 5 and Corollary 3 ).

The argument is based on considering the compact four-manifolds with harmonic curvature satisfying an additional condition on the curvature tensor, which essentially means that they are locally conformal to products of surfaces. It turns out (THEOREM 3 ) that those manifolds are parametrized, up to a local isometry, by metrics on compact surfaces with a second order condition imposed on their Gauss curvatures. The existence of such surface metrics in turn follows from a bifurcation theorem (Section 4).

I wish to thank Jerry KAzDAN and Richard Schoen for valuable suggestions.

\section{Preliminaries}

Let $(M, g)$ be a Riemannian manifold with $n=\operatorname{dim} M \geq 3$. Applying contractions to the second Bianchi identity $d R=0$ (in local coordinates, $\nabla_{q} R_{i j k \ell}+\nabla_{i} R_{j q k \ell}+\nabla_{j} R_{q i k \ell}=0$ ), we obtain the following relations :

$$
\begin{aligned}
& \delta R=-d r, \quad\left[i . e .-\nabla^{q} R_{q i j k}=-\left(\nabla_{j} r_{i k}-\nabla_{k} r_{i j}\right)\right] \\
& (n-2) \delta W=(3-n) d\left[r-(2 n-2)^{-1} u g\right]
\end{aligned}
$$

and

$$
2 \delta r=-d u, \quad\left[i . e .-2 \nabla^{k} r_{k i}=-\partial_{i} u\right] .
$$

Here $R, W, r$ and $u$ are the curvature tensor, Weyl conformal tensor, Ricci tensor and scalar curvature of $g$, respectively, with the sign conventions such that $r_{i j}=R_{i s j}{ }^{s}, u=g^{i j} r_{i j}$ and

$$
\begin{array}{r}
W_{i j k \ell}=R_{i j k \ell}-(n-2)^{-1}\left(g_{i k} r_{j \ell}+g_{j \ell} r_{i k}-g_{j k} r_{i \ell}-g_{i \ell} r_{j k}\right) \\
+((n-1)(n-2))^{-1} u\left(g_{i k} g_{j \ell}-g_{j k} g_{i \ell}\right) .
\end{array}
$$

To these tensors we apply the exterior differentiation $d$ and its formal adjoint, the divergence $\delta$, by viewing them as differential forms on $M$ valued in appropriate vector bundles.

We say that $(M, g)$ has harmonic curvature (resp., harmonic Weyl tensor) if $\delta R=0$ (resp., $\delta W=0$ ).

By a Codazzi tensor on a Riemannian manifold $(M, g)$ we mean a symetric $(0,2)$ tensor field $b$ on $M$ satisfying the Codazzi equation $d b=0$ (i.e., $\nabla_{j} b_{i k}=\nabla_{k} b_{i j}$ ). The following lemma is an obvious consequence of (1), (2) and (3) together with the fact that condition $d\left[r-(2 n-2)^{-1} u g\right]=$ 0 characterizes conformally flat manifolds in dimension $n=3[16$, p. 306].

TOME $116-1988-\mathrm{N}^{\circ} 2$ 
LEMмA 1. - Let $(M, g)$ be an $n$-dimensional Riemannian manifold, with $n \geq 3$.

(i) If $(M, g)$ has harmonic curvature, then its scalar curvature is constant and $\delta W=0$.

(ii) If $n \geq 4, \delta W=0$ and $(M, g)$ has constant scalar curvature, then $\delta R=0$.

(iii) $(M, g)$ has harmonic curvature if and only if its Ricci tensor is Codazzi.

(iv) If $n=3,(M, g)$ has harmonic curvature if and only if it is conformally flat and has constant scalar curvature.

(v) If $(M, g)$ is a Riemannian product, then it has harmonic curvature if and only if the factor manifolds do so (where, for $n<3$, by harmonic curvature we mean constant curvature).

Example 1. - The following Riemannian four-manifolds have harmonic curvature in view of LEMMA 1 . Note that each of the classes listed below contains compact manifolds.

(i) Einstein spaces.

(ii) Conformally flat manifolds with constant scalar curvature.

(iii) Four-manifolds isometrically covered by products of surfaces with constant curvatures.

(iv) Quotients of Riemannian products $\mathbb{R} \times N, N$ being a conformally flat 3-manifold with constant scalar curvature.

The classification problem for compact four-manifolds with harmonic curvature is still unsolved, even if one excludes the types listed in Example 1 . However, there are known topological obstructions for the existence of metrics with harmonic curvature on compact orientable four-manifolds $M$ : a necessary condition is

$$
2 \chi|\tau| \geq 3 \tau^{2}
$$

$\tau$ being the signature and $\chi$ the Euler characteristic of $M$. In fact, if $\tau \neq 0$, any metric with harmonic curvature on $M$ is Einstein by Bourguignon's theorem [5, Théorème 7.1], and so $M$ satisfies the THORPE inequality $2 \chi \geq 3|\tau| \quad[18]$.

Theorem 1 (D. DeTurck and H. Goldschmidt [11]). - Every Riemannian metric $g$ with harmonic curvature in the Hölder class $C^{6, \alpha}$ is analytic in suitable local coordinates.

The above assertion means that $g$ is analytic with respect to a unique analytic structure compatible with the original differentiable structure. In fact, the transition between two coordinate systems making $g$ analytic 
is analytic, since it is an isometry between analytic metrics on open Euclidean sets.

For each oriented Riemannian four-manifold $(M, g)$, the bundle $\Lambda^{2} M$ of 2-forms splits into the 3-dimensional ( \pm 1 )-eigenspace bundles $\Lambda_{+}^{2} M$, $\Lambda_{-}^{2} M$ of the Hodge star operator *. Since the Weyl tensor $W$ operating on $\Lambda^{2} M$ commutes with $*$ [17, Theorem 1.3], it gives rise to the restrictions $W^{ \pm}: \Lambda_{ \pm}^{2} M \rightarrow \Lambda_{ \pm}^{2} M$, each of which can be trivially extended to an endomorphism of $\Lambda^{2} M$, i.e., to a $(0,4)$ tensor field on $M(c f$. [1]).

Lemma 2 (cf. $[4$, p. $440,16.21])$. - Let $b$ be a Codazzi tensor on a Riemannian four-manifold $(M, g)$. Then, at each point $x \in M$ with

$$
b(x) \neq \frac{1}{4} \operatorname{trace} b(x) \cdot g(x),
$$

$W^{+}(x)$ and $W^{-}(x)$ have equal spectra, including multiplicities.

Proof. - It is sufficient to show that at any $x$ satisfying (5) there exists an orthonormal basis $X_{1}, \ldots, X_{4}$ of $T_{x} M$ which diagonalizes $W$ in the sense that

$$
W\left(X_{i} \wedge X_{j}\right)=\lambda_{i j} X_{i} \wedge X_{j}, \quad i \neq j
$$

with some numbers $\lambda_{i j}$. In fact, for a fixed local orientation and for $i, j$, $k, \ell$ mutually distinct, $*\left(X_{i} \wedge X_{j}\right)= \pm X_{k} \wedge X_{\ell}$ and, since $W$ commutes with $*$ [17, Theorem 1.3], $\lambda_{i j}=\lambda_{j i}=\lambda_{k \ell}$. Now, for some $\epsilon= \pm 1$, $\omega_{j}^{ \pm}=X_{1} \wedge X_{j} \pm \epsilon X_{k} \wedge X_{\ell} \in \Lambda_{ \pm}^{2} M$ for any cyclic permutation $(j, k, \ell)$ of $\{2,3,4\}$, and $W^{ \pm}\left(\omega_{j}^{ \pm}\right)=\lambda_{1 j} \omega_{j}^{ \pm}$, so that $W^{+}$and $W^{-}$have equal spectra at $x$.

Note that an orthonormal basis of $T_{x} M$ diagonalizing $R$ will also diagonalize $W$ in view of (4), since such a basis consists of eigenvectors of $r$.

To prove the existence of a diagonalizing basis for $W$, we may assume that the point $x$ satisfying (5) lies in the dense subset of points at which the number $m$ of distinct eigenvalues of $b$ is locally constant. Thus, near, $x, b$ has distinct eigenvalue functions $\lambda(1), \ldots, \lambda(m)$ of multiplicities $k_{1} \leq \cdots \leq k_{m}$ and four cases are possible :

(a) $m=4$;

(b) $m=2, \quad k_{1}=k_{2}=2$;

(c) $m=2, \quad k_{1}=1, \quad k_{2}=3$;

(d) $m=3, \quad k_{1}=k_{2}=1, \quad k_{3}=2$.

In cases (a) and (b), the existence of an orthonormal basis diagonalizing $R(x)$ follows from [10, Remark 2] and [10, Lemma 1]. Now assume case (c)

томе $116-1988-\mathrm{N}^{\circ} 2$ 
(resp., case (d)), and let $V_{\lambda(i)}$ be the $\lambda(i)$-eigenspace of $b(x)$. The spans $V_{\lambda(i)} \wedge V_{\lambda(j)}$ of exterior products are invariant under $R(x)$ [10, Theorem 1] and hence also under the self-adjoint endomorphism $W(x)$ of $\Lambda^{2} M$ at $x$. Thus, we can choose orthonormal eigenvectors $X_{1}, \ldots, X_{4}$ of $b(x)$ with $X_{1} \in V_{\lambda(1)}$ and $W\left(X_{1} \wedge X_{i}\right)=\mu_{i} X_{1} \wedge X_{i}$ for some $\mu_{i}, 2 \leq i \leq 4$. Since $W$ commutes with $*$, we obtain (6) with some $\lambda_{i j}$ (cf. the argument following (6)), which completes the proof.

Corollary 1 (cf. [4, p. 442, 16.28]. - Let $(M, g)$ be a Riemannian four-manifold with harmonic Weyl tensor. At any point $x$ with $r(x) \neq$ $\frac{1}{4} u(x) g(x), W^{+}(x)$ and $W^{-}(x)$ have equal spectra, including multiplicities.

Proof. - By (2), Lemma 2 can be applied to $b=r-\frac{1}{6} u g$.

Remark 1. - It is easy to verify that the Riemannian product of manifolds $\left(M_{\alpha}, g_{\alpha}\right)$ with $\operatorname{dim} M_{\alpha}=n_{\alpha}$ having sectional curvatures $K_{\alpha}$, $\alpha=1,2$ (where $K_{\alpha}=0$ if $n_{\alpha}=1$ ) is conformally flat if and only if both $K_{1}, K_{2}$ are constant and $\left(n_{1}-1\right)\left(n_{2}-1\right)\left(K_{1}+K_{2}\right)=0$.

In the sequel we shall often consider oriented Riemannian fourmanifolds $(M, g)$ with the following property :

(7) The endomorphism $W^{+}$of $\Lambda_{+}^{2} M$ has less than 3 distinct eigenvalues at each point.

Example 2.

(i) Every Kähler manifold of real dimension four satisfies (7) for the natural orientation ([12], [14] and [9, Proposition 2]).

(ii) Condition (7) is conformally invariant.

(iii) Any Riemannian four-manifold locally conformal to a product of surfaces satisfies (7) for either local orientation. In fact, a product of surfaces is locally Kähler for two complex structures, corresponding to different orientations.

We shall now construct examples of Riemannian four-manifolds with $\delta W=0$ and (7). According to Theorem 2 below, these are, essentially, the only examples of this type.

Example 3. - Let $\left(N_{\alpha}, h_{\alpha}\right)$ be Riemannian surfaces with Gauss curvatures $K_{\alpha}, \alpha=1,2$. The metric

$$
g=\left(K_{1}+K_{2}\right)^{-2}\left(h_{1} \times h_{2}\right),
$$

defined wherever $K_{1}+K_{2} \neq 0$ in $N_{1} \times N_{2}$, has harmonic Weyl tensor, which is non-zero everywhere, and satisfies (7) for either local orientation. Denoting by $|W|$ the $g$-norm of the Weyl tensor $W$ of $g$, so that

$$
|W|^{2}=\frac{1}{4} g^{i m} g^{j p} g^{k q} g^{\ell s} W_{i j k \ell} W_{m p q s}
$$


we have :

$$
h_{1} \times h_{2}=3^{1 / 3}|W|^{2 / 3} g .
$$

In fact, the norm of the Weyl tensor of $h_{1} \times h_{2}$ is easily verified to be $3^{-1 / 2}\left|K_{1}+K_{2}\right|$ so that we obtain (10) from the conformal invariance of $W$ and $W \neq 0$ wherever $K_{1}+K_{2} \neq 0$. In view of Example 2 (iii), $g$ satisfies (7) for either local orientation. Finally, for any Kähler metric $g^{\prime}$ in dimension four, the metric $\left(u^{\prime}\right)^{-2} g^{\prime}$, defined wherever $g^{\prime}$ has nonzero scalar curvature $u^{\prime}$, satisfies $\delta W^{+}=0$ for the natural orientation [9, Proposition 3]. Since $g^{\prime}=h_{1} \times h_{2}$ is locally Kähler for both orientations and $u^{\prime}=2\left(K_{1}+K_{2}\right)$, condition $\delta W=0$ holds for $g$.

TheOREM 2 ( $c f .[4$, p. 443, 16.32]. - Let $(M, g)$ be an oriented Riemannian four-manifold satisfying $\delta W=0$ and (7). In a neighborhood of each point $x$ with $W(x) \neq 0$ and $r(x) \neq \frac{1}{4} u(x) g(x), g$ has the form (8) for suitable surface metrics $h_{1}, h_{2}$ and $g$ determines $h_{1} \times h_{2}$ by (10).

Proof. - By Corollary 1, $\left|W^{+}\right|^{2}=\left|W^{-}\right|^{2}=\frac{1}{2}|W|^{2}>0$ near $x$. As $\delta W=\delta W^{+}+\delta W^{-}$is an orthogonal decomposition, $\delta W^{+}=\delta W^{-}=0$. Applying [9, Proposition 5] to either orientation ( $c f$. Corollary 1) we see that the metric $g^{\prime}=3^{1 / 3}|W|^{2 / 3} g$, defined near $x$, is Kähler for two complex structures $J^{+}, J^{-}$, corresponding to different orientations. Viewed as 2forms, $J^{ \pm}$are local sections of $\Lambda_{ \pm}^{2} M$ (see, e.g., [9, Lemma 2 (ii)]) and so $A=J^{+} \circ J^{-}$is a $g^{\prime}$-parallel self-adjoint $(1,1)$ tensor field, the eigenspaces of which are $g^{\prime}$-parallel plane fields [9, Lemma 2 (iv)]. Thus, near $x$, $g^{\prime}=h_{1} \times h_{2}$ for some surface metrics $h_{1}, h_{2}$. Now (8) follows from the conformal invariance of $W$ with the fact that, for $h_{1} \times h_{2},|W|$ is proportional to $\left|K_{1}+K_{2}\right|$ (cf. Example 3). This completes the proof.

We can now determine which of the metrics described in Example 3 have harmonic curvature (which here means constant scalar curvature, $c f$. LEMMA 1).

LEMMA 3. - Let $\left(N_{\alpha}, h_{\alpha}\right)$ and $g$ be as in Example 3.

(i) If $g$ has harmonic curvature and non-parallel Ricci tensor, then the following condition is satisfied:

(*) One of the surface metrics has constant curvature $c$ (for instance, $\left.K_{2}=c\right)$, while the other metric $h=h_{1}$ has non-constant curvature $K=K_{1}$ such that $(K+c)^{3}-3(K+c) \Delta K-6|d K|^{2}=\lambda^{3}$ for $a$ constant $\lambda$, where $\Delta=-h^{i j} \nabla_{i} \nabla_{j}$ and ||$^{2}$ refer to the geometry of $h$.

(ii) If $\left(^{*}\right)$ holds, $g$ has harmonic curvature.

Proof. - For conformally related metrics $h$ and $g=\Phi^{-2} h$ in dimension $n, \Phi>0$, the scalar curvature $u_{g}$ of $g$ is given by

$$
u_{g}=u \Phi^{2}-2(n-1) \Phi \Delta \Phi-n(n-1)|d \Phi|^{2},
$$

томе $116-1988-\mathrm{N}^{\circ} 2$ 
where $u,||$ and $\Delta=-h^{i j} \nabla_{i} \nabla_{j}$ correspond to $h$. If $n=4, h=h_{1} \times h_{2}$ and $\Phi=K_{1}+K_{2}$, this gives

$$
\frac{1}{2} u_{g}=\left(K_{1}+K_{2}\right)^{2}-3\left(K_{1}+K_{2}\right)\left(\Delta K_{1}+\Delta K_{2}\right)-6\left(\left|d K_{1}\right|^{2}+\left|d K_{2}\right|^{2}\right),
$$

$\Delta$ and $\mid$ being the Laplacian and the norm corresponding to either surface metric $h_{\alpha}$. Now, if $\left(^{*}\right)$ holds, $u_{g}=2 \lambda^{3}$ is constant, so that $g$ has harmonic curvature in view of Example 3 and Lemma 1 (ii), which proves (ii).

To prove (i), assume that $g$ has harmonic curvature and so $u_{g}$ is constant by Lemma $1(\mathrm{i})$. Suppose first that $K_{1}, K_{2}$ are both non-constant. For any $x \in N_{1}$, pick $y \in N_{2}$ with $d K_{2}(y) \neq 0$ and $K_{1}(x)+K_{2}(y) \neq 0$. For a curve through $y, t=K_{2}$ can be used as parameter. Thus, wherever $K_{1}+t \neq 0$ in $N_{1}$, we have from (11)

$$
\frac{1}{2} u_{g}=\left(K_{1}+t\right)^{3}-3\left(K_{1}+t\right)\left(\Delta K_{1}+\alpha(t)\right)-6\left|d K_{1}\right|^{2}-6 \beta(t)
$$

with some numbers $\alpha(t), \beta(t)$. Differentiating (12) with respect to $t$ we obtain

$$
0=3\left(K_{1}+t\right)^{2}-3\left(\Delta K_{1}+\alpha(t)\right)-3 \alpha^{\prime}(t)\left(K_{1}+t\right)-6 \beta^{\prime}(t) .
$$

This implies that $\Delta K_{1}$ is a second order polynomial in $K_{1}$ with leading coefficient 1 and so, by (12), $\left|d K_{1}\right|^{2}$ is a third order polynomial in $K_{1}$ with leading coefficient $-\frac{1}{3}$. For reasons of symmetry, the same holds for $K_{2}$, and using (11) we easily conclude that, for $\alpha=1,2$ :

$$
\begin{aligned}
\Delta K_{\alpha} & =K_{\alpha}^{2}+2 p_{\alpha} K_{\alpha}-q_{\alpha} \\
\left|d K_{\alpha}\right|^{2} & =s_{\alpha}+t K_{\alpha}-p_{\alpha} K_{\alpha}^{2}-\frac{1}{3} K_{\alpha}^{3}
\end{aligned}
$$

with constants $p_{\alpha}, q_{\alpha}, s_{\alpha}, v$ such that $p_{1}+p_{2}=0$ and $q_{1}+q_{2}=2 v$. Using the same argument as in Remark 4 below, we conclude that $p_{\alpha}=0$ and $q_{\alpha}=v$ and that the tensor $T_{\alpha}=2 \nabla d K_{\alpha}+\left(K_{\alpha}^{2}-v\right) h_{\alpha}$ vanishes wherever $d K_{\alpha} \neq 0, \nabla$ being the Riemannian connection of $h_{\alpha}$. From (13) with a continuity argument, we see that $T_{\alpha}=0$ everywhere in $N_{\alpha}$.

For conformally related metrics $h$ and $g=\Phi^{-2} h$ in dimension $n$, the Ricci tensor $r_{g}$ of $g$ is given by

$$
r_{g}=r+(n-2) \Phi^{-1} \nabla d \Phi-\left(\Phi^{-1} \Delta \Phi+(n-1) \Phi^{-2}|d \Phi|^{2}\right) h,
$$

where $r, \nabla, \Delta=-h^{i j} \nabla_{i} \nabla_{j}$ and || correspond to $h$. For $n=4, h=h_{1} \times h_{2}$ and $\Phi=K_{1}+K_{2}$, formula (15) with $T_{\alpha}=0$ implies that, at each point, $r_{g}$ has two double eigenvalues (which may coincide). As $r_{g}$ is a 
Codazzi tensor with respect to $g$ and has constant $g$-trace (LEMMA 1), it follows from [8, Lemma 3] that $r_{g}$ is $g$-parallel. This proves that if $g$ has harmonic curvature and non-parallel Ricci tensor, $K_{1}$ or $K_{2}$ is constant, which implies $(*)$ in view of $(11)$.

According to Lemma 3, certain Riemannian surfaces can be used to construct four-manifolds with harmonic curvature, which are not conformally flat (Example 3). To make sure that this construction can really give new examples of such manifolds, we have to determine which of those manifolds are Einstein or locally reducible.

LEMmA 4. - Let $(N, h),\left(N_{c}, h_{c}\right)$ be Riemannian surfaces such that $h_{c}$ has constant curvature $c \neq 0$. Consider the metric

$$
g=(K+c)^{-2}\left(h \times h_{c}\right)
$$

defined in the open subset $U$ of $N \times N_{c}$ where $K+c \neq 0, K$ being the Gauss curvature of $h$.

(i) If $g$ admits a non-trivial parallel vector field in a neighborhood of each point of $U$, then the metric $(K+c)^{-2} h$, defined wherever $K+c \neq 0$ in $N$, is flat.

(ii) Suppose that $N$ is oriented and

$$
(K+c)^{3}-3(K+c) \Delta K-6|d K|^{2}=\lambda^{3}
$$

for a constant $\lambda$, where $\Delta=-h^{i j} \nabla_{i} \nabla_{j}$ and || refer to $h$. If the Ricci tensor of $g$ is $g$-parallel, then $J(\nabla K)$ is a Killing field on $(N, h)$, where $J$ is the complex structure of $(N, h)$ and $\nabla K$ is the gradient of $K$.

Proof.

(i) Let $X \neq 0$ be a local $g$-parallel vector field. Hence $L_{X} g=0$, so that the local flow of $X$ preserves the norm of the Weyl tensor of $g$. Therefore $(c f .(8),(10))$ it also preserves $K$, viewed as a function on $N \times N_{c}$, i.e. $d K(X)=0$. The projections of $X$ onto the $N$ and $N_{c}$ factors of $N \times N_{c}$ give local vector fields on $N, N_{c}$, each parametrized by a subset of the other surface. Since $X$ is $g$-parallel and $d K(X)=0$, it is easy to see that each $N_{c}$-projection of $X$ is $h_{c}$-parallel. As $c \neq 0, X$ is tangent to $N$ at each point. The $N$-factors are totally geodesic in $(U, g)$, so that $X$, restricted to each factor, is parallel for the induced metric $(K+c)^{-2} h$. Therefore,this metric is flat.

(ii) Using (15) with $n=4, h$ replaced by $h \times h_{c}$ and $\Phi=K+c$ we see that the $N$ and $N_{c}$ factor foliations of $U$ are $r_{g}$-orthogonal and $r_{g}$ restricted to them is given by

$$
\begin{aligned}
\left(r_{g}\right)_{N}= & K h+2(K+c)^{-1} \nabla d K \\
& -(K+c)^{-2}\left[(K+c) \Delta K+3|d K|^{2}\right] h \\
\left(r_{g}\right)_{N_{c}}= & \left(c \tau^{2}-\tau \Delta \tau-3|d \tau|^{2}\right)(g)_{N_{c}}
\end{aligned}
$$

томе $116-1988-\mathrm{N}^{0} 2$ 
where $\tau=K+c$. Thus, $c \tau^{2}-\tau \Delta \tau-3|d \tau|^{2}$ is an eigenvalue of $r_{g}$ with the eigenspace containing $T N_{c}$ at each point, so that, if $r_{g}$ is $g$-parallel,

$$
c \tau^{2}-\tau \Delta \tau-3|d \tau|^{2}=\mu
$$

for a constant $\mu$. Now suppose that one of the eigenvalues of $r_{g}$ is simple. Since $r_{g}$ is $g$-parallel, $g$ admits a local non-zero parallel vector field and, by (i), the curvature $\tau^{3}-c \tau^{2}-\tau \Delta \tau-|d \tau|^{2}$ of the metric $\tau^{-2} h$ is zero wherever $\tau=K+c \neq 0$. By continuity, we have in $(N, h)$

$$
\tau^{3}-c \tau^{2}-\tau \Delta \tau-|d \tau|^{2}=0
$$

By the hypothesis of (ii), $\tau^{3}-3 \tau \Delta \tau-6|d \tau|^{2}=\lambda^{3}$ which, together with (17) and (18) easily implies that $\tau$ is constant. Hence our assertion follows if $r_{g}$ has a simple eigenvalue. In the remaining case all eigenvalues of $r_{g}$ are multiple and so $\left(r_{g}\right)_{N}$ is a constant multiple of $(g)_{N}$. In view of (16), this implies that $\nabla d K=\psi h$ wherever $K+c \neq 0$ in $N$, and, by continuity, also at points where $K+c=0, \psi$ being a function on $N$. Now one easily verifies that $J(\nabla K)$ is a Killing field.

Proposition 1. - Let an oriented Riemannian four-manifold $(M, g)$ with harmonic curvature satisfy (7). Then either $W^{+}=0$ identically, or $W^{+} \neq 0$ everywhere in $M$.

Proof. - Suppose first that $(M, g)$ has parallel Ricci tensor, If it is Einstein, our assertion follows from [9, Proposition 5 (iv)]. Otherwise it is locally a product of lower dimensional Einstein spaces, so that $g$ is locally symmetric and $W^{+}$is parallel, which gives our assertion.

Now we may assume that $W \neq 0$ somewhere and $r$ is not parallel. Fix any $x_{0} \in M$ and choose $\epsilon>0$ such that, for all $y$ in the open $\epsilon$-ball $B$ around $x_{0}$, exp is defined on the whole closed $\epsilon$-ball with center 0 in $T_{y} M$. The function $F=6\left|W^{+}\right|^{2}$ on $M$ is not identically zero (Corollary 1) and it is analytic (Theorem 1), so, unless it is constant (which implies our assertion), it has a regular value $t_{0} \neq 0, t_{0} \neq F\left(x_{0}\right)$ attained in $B$. Choose $y_{0}$ in the closed subset $F^{-1}\left(t_{0}\right)$, minimizing its distance from $x_{0}$ and the corresponding geodesic $\gamma$ from $y_{0}$ to $x_{0}$ (which is possible even if $g$ is not complete). Near $y_{0}$, the metric $g^{\prime}=F^{1 / 3} g$ is a product of surface metrics $h, h_{c}$, which gives rise to mutually orthogonal foliations $\mathcal{F}, \mathcal{F}_{c}$, where $h_{c}$ has constant curvature $c$ and $g=(K+c)^{-2}\left(h \times h_{c}\right), K$ being the Gauss curvature of $h$ (Lemma 1 (i), Theorem 2, Corollary 1, Theorem 1 , Lemma 3 (i)). Choose an open simply connected subset $U$ of the leaf of $\mathcal{F}_{c}$ through $y_{0}$ with $y_{0} \in U \subset B$ and $g$-orthonormal sections $X, Y$ of the normal bundle of $U$, parallel with respect to the normal connection 
determined by $g^{\prime}$ (which is possible, since $K+c$ is constant along $U$ ). Let $D_{\epsilon}$ be the disc with center 0 and radius $\epsilon$ in $\mathbb{R}^{2}$. The analytic mapping

$$
\Phi: D_{\epsilon} \times U \rightarrow M \quad \text { with } \Phi((s, t), y)=\exp _{y}(s X(y)+t Y(y))
$$

where exp is the exponential mapping of $g$, is well-defined by our choice of $\epsilon$. Since $K+c$ is constant along $\mathcal{F}_{c}$, the leaves of $\mathcal{F}$ are totally geodesic in $(M, g)$ and so, in a neighborhood of $\{0\} \times U$ in $D_{\epsilon} \times U$ the pull-back $\Phi^{*}\left(F^{1 / 3} g\right)=\left(F^{1 / 3} \circ \Phi\right) \Phi^{*} g$ is a product metric in a manner compatible with the decomposition of $D_{\epsilon} \times U$, that is,

$$
\left(F^{1 / 3} \circ \Phi\right) \Phi^{*} g=h^{\prime} \times h_{c}^{\prime}
$$

for some metric $h_{c}^{\prime}$ on $U$ and a metric $h^{\prime}$ defined near 0 in $D_{\epsilon}$. By Theorem 1 , the restriction of $\left(F^{1 / 3} \circ \Phi\right) \Phi^{*} g$ to the $U$-tangent subbundle of $T\left(D_{\epsilon} \times U\right)$ is "constant along $D_{\epsilon}$ " and equal to $h_{c}^{\prime}$, since this is the case near $\{0\} \times U$. Consequently, $F^{1 / 3} \circ \Phi \neq 0$ everywhere in $D_{\epsilon} \times U$. The geodesic $\gamma$ from $y_{0}$ to $x_{0}$ was chosen so that it is perpendicular to $F^{-1}\left(t_{0}\right) \supset U$ at $y_{0}$ and has length $L(\gamma)<\epsilon$. Therefore $x_{0} \in \Phi\left(D_{\epsilon} \times U\right)$ and so $F\left(x_{0}\right) \neq 0$. Since $x_{0}$ was arbitrary, this completes the proof.

LEMMA 5. - Let $\tau$ be an everywhere non-zero non-constant function on a compact Riemannian manifold $(M, g)$, such that

$$
\tau^{3}-3 \tau \Delta \tau-6|d \tau|^{2}=\lambda^{3}
$$

for a constant $\lambda$, where $\Delta=-g^{i j} \nabla_{i} \nabla_{j}$. Then $\tau>0$ and $\lambda>0$.

Proof. - Fix $x, y \in M$ with $\tau(x)=\max \tau>\min \tau=\tau(y)$. If $\tau$ was negative, we would have $\lambda^{3}=\tau^{3}(y)-3 \tau(y) \Delta \tau(y) \leq \tau^{3}(y)<\tau^{3}(x) \leq$ $\tau^{3}(x)-3 \tau(x) \Delta \tau(x)=\lambda^{3}$. This contradiction shows that $\tau>0$ and so $\lambda^{3}=\tau^{3}(y)-3 \tau(y) \Delta \tau(y) \geq \tau^{3}(y)>0$.

LEMMA 6. - Let a compact surface $(N, h)$ with non-constant Gauss curvature $K$ satisfy $|K+c|>0$ and $(K+c)^{3}-3(K+c) \Delta K-6|d K|^{2}=\lambda^{3}$ for some constants $c, \lambda$. Then $K+c>0, \lambda>0$ and $c>0$.

Proof. $-K+c>0$ and $\lambda>0$ in view of Lemma 5 . Integrating by parts over $N$ we obtain, with $\tau=K+c$,

$$
\begin{gathered}
3 \int \tau^{-4}|d \tau|^{2} \Delta \tau=\int \tau^{-3}\left[\langle d \tau, d \Delta \tau\rangle-(\Delta \tau)^{2}\right] \\
3 \int \tau^{-4}\left\langle d \tau, d|d \tau|^{2}\right\rangle=2 \int \tau^{-3}\left[(\tau-c)|d \tau|^{2}-\langle d \tau, d \Delta \tau\rangle+|\nabla d \tau|^{2}\right] \\
\text { тОМе } 116-1988-\mathrm{N}^{\mathrm{o}} 2
\end{gathered}
$$


where, in the second formula, we have used the Ricci-Weitzenböck identity $\nabla^{i} \nabla_{i} \nabla_{j} \tau=K \partial_{j} \tau-\partial_{j} \Delta \tau$. Now, we have

$$
\begin{aligned}
0 & =\int \tau^{-4}\left\langle d \tau, d\left[\tau^{3}-3 \tau \Delta \tau-6|d \tau|^{2}\right]\right\rangle \\
=3 \int \tau^{-2}|d \tau|^{2}-3 \int \tau^{-4}|d \tau|^{2} \Delta \tau-3 \int \tau^{-3}\langle d \tau, d \Delta \tau\rangle & -6 \int \tau^{-4}\left\langle d \tau, d|d \tau|^{2}\right\rangle \\
& -6
\end{aligned}
$$

so that, from the preceding equalities,

$$
0=\int \tau^{-3}\left[(4 c-\tau)|d \tau|^{2}+(\Delta \tau)^{2}-4|\nabla d \tau|^{2}\right]
$$

Suppose that $c \leq 0$. As $\tau>0,4 c-\tau<4 c \leq 0$ which, by the Schwarz inequality $(\Delta \tau)^{2} \leq 2|\nabla d \tau|^{2}$, would contradict the last relation by making the integral negative. This contradiction completes the proof.

The following fact is well known.

LEMMA 7. - Let a Riemannian surface $(N, h)$ admit a $C^{\infty}$ function $\tau$ such that $|d \tau|^{2}$ and $\Delta \tau$ are functions of $\tau$. In a neighborhood of each point where $d \tau \neq 0,(N, h)$ admits a non-trivial Killing field, the local flow of which leaves $\tau$ invariant. This Killing field is unique up to a constant factor.

Proof. - Let $d \tau\left(x_{0}\right) \neq 0$. Near $x_{0}$ there exist coordinates $s, t$ such that $s$ is constant along the levels of $\tau$, while $t$ is constant along the integral curves of $\nabla \tau$. Hence $\tau=\tau(s)$ and $h=e^{\alpha(s, t)} d s^{2}+e^{2 \beta(s, t)} d t^{2}$ for some functions $\alpha, \beta$. Now $|d \tau|^{2}=e^{-\alpha(s, t)}\left(\tau^{\prime}(s)\right)^{2}$ is a function of $s$ and hence so is $\alpha$, so that we may assume $\alpha=0$, replacing $s$ by a suitable function of $s$. On the other hand,

$$
\Delta \tau=-h^{i j} \nabla_{i} \nabla_{j} \tau=-\tau^{\prime \prime}(s)-\tau^{\prime}(s) \frac{\partial}{\partial s} \beta(s, t)
$$

is a function of $s$, i.e., $\beta(s, t)=\Phi(s)+\psi(t)$ and we may assume $\psi=0$, replacing $t$ by a function of $t$. Thus, $h=d s^{2}+e^{2 \Phi(s)} d t^{2}$ and the required Killing field corresponds to the local flow of $t$-translations. The uniqueness assertion is due to the fact that a non-constant function multiplied by a non-trivial Killing field never gives a Killing field again. 


\section{A classification theorem}

We already know (Theorem 2 and Lemma 3) that Riemannian fourmanifolds with non-parallel Ricci tensor, which are not conformally flat and satisfy (7), can, locally, be obtained from certain Riemannian surfaces by an explicit construction. We shall now prove the global version of this fact for compact manifolds.

Example 4. - Suppose that we are given a compact oriented Riemannian surface $(N, h)$ with non-constant Gauss curvature $K$ such that

$$
K+c>0
$$

and

$$
(K+c)^{3}-3(K+c) \Delta K-6|d K|^{2}=\lambda^{3}
$$

with real $c, \lambda>0$, where $\Delta=-h^{i j} \nabla_{i} \nabla_{j}$ (see Example 5 and Section 4 for existence results), a 2-sphere $\left(S^{2}, h_{c}\right)$ of constant curvature $c$ and a homomorphism $\sigma: \pi_{1} N \rightarrow \mathrm{SO}(3)$. The formula

$$
\Phi \cdot(x, \dot{y})=(\Phi x, \sigma(\Phi) y)
$$

defines a properly discontinuous action of $\pi_{1} N$ on $\widetilde{N} \times S^{2}$ leaving the metric $\widetilde{g}=(\widetilde{K}+c)^{-2}\left(\widetilde{h} \times h_{c}\right)$ invariant, where $(\widetilde{N}, \widetilde{h})$ is the Riemannian universal covering of $(N, h)$ with Gauss curvature $\widetilde{K}$ and $\Phi \in \pi_{1} N$ acts on $\tilde{N}$ as a deck transformation. The quotient

$$
(M, g)=\left(\tilde{N} \times S^{2}, \widetilde{g}\right) / \pi_{1} N
$$

then is a compact orientable Riemannian four-manifold with harmonic curvature, which is not conformally flat, has non-parallel Ricci tensor and satisfies (7) for either orientation. Moreover, the metric conformal to $g$ and locally isometric to $h \times h_{c}$ is given in terms of $g$ by (10). In fact, in view of Lemma 3 (ii) and Example 3 we only need to show that the Ricci tensor of $g$ is not parallel. However, if it was parallel, by LEMMA 4 (ii) $\nabla K$ would be a holomorphic vector field on $(N, h)$, i.e., $h$ would be an extremal Kähler metric. As shown by E. CALABI $[7$, p. 276] that is impossible unless $K$ is constant.

Remark 2. - A Riemannian metric $h$ of class $C^{9}$ on a surface $N$, satisfying $|K+c|>0$ and (21) for some real $c, \lambda$ is necessarily analytic (in suitable local coordinates). In fact, by Lemma 3 (ii), $g=(K+c)^{-2}\left(h \times h_{c}\right)$ then is a $C^{7}$ metric with harmonic curvature on $M=N \times N_{c},\left(N_{c}, h_{c}\right)$ being a surface of constant curvature $c$. Thus, $g$ is analytic by THEOREM 1

$$
\text { TOME } 116-1988-\mathrm{N}^{\mathrm{O}} 2
$$


and hence so is the metric $h \times h_{c}=3^{1 / 3}|W|^{2 / 3} g$ (cf. Example 3), so that the totally geodesic submanifold $(N, h)$ of $\left(M, h \times h_{c}\right)$ is analytic, too.

TheOREM 3. - Let $(M, g)$ be a compact, oriented, non-conformallyflat Riemannian four-manifold with harmonic curvature and with nonparallel Ricci tensor, such that the endomorphism $W^{+}$of $\Lambda_{+}^{2} M$ has, at each point, less than three distinct eigenvalues.

Then, up to a two-fold Riemannian covering, $(M, g)$ is obtained as in Example 4 from a compact oriented Riemannian surface $(N, h)$ with nonconstant Gauss curvature $K$ satisfying (20) and (21) for some real $c, \lambda>0$ together with a homomorphism $\sigma: \pi_{1} N \rightarrow \mathrm{SO}(3)$.

In particular, up to a two-fold cover $M$ is the total space of an $\mathrm{SO}(3)$ bundle with fibre $S^{2}$ over $N$, admitting a flat connection, and the metric $|W|^{2 / 3} g$, defined everywhere in $M$ is, locally, a product of surface metrics, one of which has positive constant curvature.

Proof. - In view of Theorem 1 and Corollary $1, W^{+}$does not vanish identically, so by Proposition 1 the metric $g^{\prime}=3^{1 / 3}|W|^{2 / 3} g$ is defined everywhere in $M$. According to Theorem 2 and Lemma $3 g^{\prime}$ is, locally, the product of surface metrics, $h, h_{c}$ satisfying $\left(^{*}\right)$ in the sense that $h_{c}$ has constant curvature $c$ and (21) holds for $h$. Since

$$
|K+c|=3^{1 / 6}|W|^{1 / 3}>0
$$

(cf. (8) and (10)), we can apply Lemma 5 to $\tau=K+c$ on the compact manifold $\left(M, g^{\prime}\right)$, concluding that $K+c>0$ everywhere and $\lambda>0$.

Let $\mathcal{F}, \mathcal{F}_{c}$ be the mutually orthogonal 2-dimensional foliations of $M$, corresponding to the local product decomposition $g^{\prime}=h \times h_{c}$. We claim that $c>0$. In fact, since $K+c>0$ on $M$, we have $K+c \geq \epsilon$ for some $\epsilon>0$ and, if we had $c \leq 0$, each leaf of $\mathcal{F}$ with the metric $h$ induced from $g^{\prime}$ would have Gauss curvature $K \geq \epsilon-c \geq \epsilon>0$, and so it would be compact by the MYERs theorem.

Now, LEMma 6 applied to the leaf would give $c>0$. This contradiction shows that $c>0$. By passing to a two-fold isometric cover of $M$ if necessary, we may assume that $\mathcal{F}_{c}$ is oriented. Since the leaves of $\mathcal{F}_{c}$ with the metric induced from $g^{\prime}$ have constant curvature $c>0$, they are 2 -spheres with trivial leaf holonomy. Hence the leaf space $N=M / \mathcal{F}_{c}$ is a compact oriented analytic surface ( $c f$. ThEorem 1 ) and the bundle projection $\psi:\left(M, g^{\prime}\right) \rightarrow N$ with fibre $S^{2}$ becomes a Riemannian submersion for a suitable metric on $N$, which we also denote by $h$ (since $\psi$ is, locally, the projection of a Riemannian product onto a factor). The restriction of $\psi$ to any leaf of $\mathcal{F}$ with the metric induced from $g^{\prime}$ is an isometric covering onto $(N, h)$. Thus, $(N, h)$ is as in Example 4. 
Pulling back the bundle $\psi: M \rightarrow N$ to the universal cover $\tilde{N}$ of $N$, we obtain a bundle $\widetilde{\psi}: \widetilde{M} \rightarrow \widetilde{N}$ with fibre $S^{2}$ such that the bundle map $\widetilde{M} \rightarrow M$ is a covering. Let $\widetilde{g}, \widetilde{g}^{\prime}, \widetilde{\mathcal{F}}, \widetilde{h}$ be the pull-backs of $g, g^{\prime}, \mathcal{F}, h$, respectively, to $\widetilde{M}$ or $\widetilde{N}$. As before, $\widetilde{\psi}$ restricted to any leaf of $\widetilde{\mathcal{F}}$ with the metric induced from $\widetilde{g}^{\prime}$ is a Riemannian covering onto $(\tilde{N}, \widetilde{h})$ and so it is a diffeomorphism, which implies that the bundle $\widetilde{\psi}: \widetilde{M} \rightarrow \widetilde{N}$ is trivial. Thus, $\widetilde{M}$ is simply connected and the locally reducible metric $\widetilde{g}^{\prime}$ is globally isometric to $h \times h_{c}$ on $\widetilde{N} \times S^{2}$. The deck transformation group $\pi_{1} N$ now can be identified with $\pi_{1} M$, acting on $\widetilde{M}$ by $\widetilde{g}^{\prime}$-isometries and making $\widetilde{\psi}$ equivariant. Each of these $\widetilde{g}^{\prime}$-isometries obviously is a product map. Assigning to it its second part which lies in $\mathrm{SO}(3)$, we obtain the required homomorphism $\sigma$. This completes the proof.

Remark 3. - Any manifold $(M, g)$ satisfying the hypotheses of ThEOREM 3 has the following property :

(**) All orbits of the isometry group $G$ of the Riemannian universal covering $(\widetilde{M}, \widetilde{g})$ of $(M, g)$ are of dimensions less than $\operatorname{dim} G$,

which is due to $\mathrm{SO}(3)$ acting on the $S^{2}$ factor of $\widetilde{M}$. However, condition $\left({ }^{*}\right)$ (in dimension 4) implies (7) for either orientation [9, Lemma 9], so that THEOREM 3 can be viewed as a classification result for compact fourmanifolds with harmonic curvature, satisfying $\left({ }^{* *}\right)$ and having $W \neq 0$ and $\nabla r \neq 0$ somewhere.

Remark 4. - Suppose that a Riemannian surface $(N, h)$ satisfies $(21)$ with two distinct pairs of constants $c, \lambda$ and $c^{\prime}, \lambda^{\prime}$ (and so $(N, h)$ is analytic by Remark 2 , since $\left.|K+c|+\left|K+c^{\prime}\right|>0\right)$. Subtracting these equalities, we see that

$$
\Delta K=F(K)=K^{2}+2 p K-q
$$

and

$$
|d K|^{2}=G(K)=s+t K-p K^{2}-\frac{1}{3} K^{3}
$$

for some constants $p, q, s, t$, so that

$$
\nabla d K=G(K)^{-1}\left[F(K)+G^{\prime}(K)\right] d K \otimes d K-\left[F(K)+\frac{1}{2} G^{\prime}(K)\right] h .
$$

The Ricci-Weitzenböck identity $\nabla^{j} \nabla_{j} \nabla_{i} K=K \partial_{i} K-\partial_{i} \Delta K$ now easily implies that $p=0$ and $t=q$ unless $K$ is constant, i.e.,

$$
2 \nabla d K+\left(K^{2}-q\right) h=0 .
$$

Therefore, for a surface $(N, h)$ with $(21)$ which does not satisfy $(22)$ with any real $q$, the constants $c, \lambda$ are determined by the local geometry of $h$. On

TOME $116-1988-\mathrm{N}^{\circ} 2$ 
the other hand, if $N$ is oriented, (22) implies that $J(\nabla K)$ is a holomorphic Killing field, $J$ being the complex structure of $(N, h)$. Consequently, by a result of E. CALABI $[7$, p. 276], a compact surface $(N, h)$ does not satisfy (22) unless $K$ is constant. If $(N, h)$ satisfies $(21)$ then, for the metric $a^{-1} h$ with a constant $a>0,(21)$ still holds if we replace $c, \lambda$ by $a c$ and $a \lambda$. Thus, if e.g. $\lambda \neq 0, c / \lambda$ is a local homothety invariant of the compact surface $(N, h)$ with non-constant curvature satisfying $(21)$. This invariant can be topologically significant, as the following lemma shows.

LEMмA 8. - Let the Gauss curvature $K$ of a compact Riemannian surface $(N, h)$ satisfy the condition

$$
(K+c)^{3}-3(K+c) \Delta K-6|d K|^{2}=\lambda^{3}
$$

with real constants $c, \lambda$, where $\Delta=-h^{i j} \nabla_{i} \nabla_{j}$. If $\chi(N) \leq 0$, then $c>\lambda$ unless $h$ is flat.

Proof. - Set $\tau=K+c$. Multiplying the equality

$$
(K+c)^{3}-\lambda^{3}-3(K+c) \Delta K-6|d K|^{2}=0
$$

by $\left(\tau^{2}+\lambda \tau+\lambda^{2}\right)^{-1}$ and integrating by parts, we obtain from the GaussBonnet formula :

$$
(c-\lambda) \operatorname{Area}(N, h)=3 \int_{N} \frac{\tau^{2}+2 \lambda \tau+3 \lambda^{2}}{\left(\tau^{2}+\lambda \tau+\lambda^{2}\right)^{2}}|d K|^{2}-2 \pi \chi(N) .
$$

Since the integral term is positive unless $K$ is constant, our assertion follows.

The existence of compact surfaces $(N, h)$ having the properties stated in Example 4 will be discussed in Section 4 . Now we shall describe those among them for which the corresponding four-manifolds with harmonic curvature are of type Example 1 (iv).

Example 5. - Let $c>\lambda>0$. The equation

$$
3(d \tau / d s)^{2}=\tau^{2}\left(3 c \tau^{2}-2 \tau^{3}-\lambda^{3}\right)
$$

has a non-constant, positive, periodic analytic solution $\tau$ on $\mathbb{R}$, which is unique up to translations of the variable $s$. In fact, the polynomial $Q(z)=3 c z^{2}-2 z^{3}-\lambda^{3}$ has 3 simple real roots, two of which are positive, as

$$
Q(-c / 2)>0, \quad Q(0)<0, \quad Q(c)>0, \quad Q(3 c / 2)<0 .
$$

For such $\tau$, the metric $h=\tau^{2}(s)\left(d s^{2}+d t^{2}\right)$ on $\mathbb{R}^{2}$ with coordinates $s, t$ has the non-constant Gauss curvature $K(s, t)=\tau(s)-c$ and satisfies (20) 
and (21), which is easily verified using the conformal transformation rule for $K$ and $\Delta$, and noting that

$$
3 \frac{d^{2} \tau}{d s^{2}}=6 c \tau^{3}-5 \tau^{4}-\lambda^{3} \tau .
$$

All $t$-translations of $\mathbb{R}^{2}$ and the $s$-translations by multiples of the period of $\tau$ leave $h$ invariant. Thus, using any lattice subgroup $\Gamma$ of $\mathbb{R}^{2}$ generated by suitable combinations of two such translations, we can project $h$ onto a metric on the torus $\mathbb{R}^{2} / \Gamma$, also denoted by $h$, representing any prescribed conformal equivalence class and having non-constant Gauss curvature for which (20) and (21) are satisfied.

Moreover, that metric is invariant under a free action of $S^{1}$ on $\mathbb{R}^{2} / \Gamma$. However, the compact four-manifolds $(M, g)$ with harmonic curvature obtained from such $(N, h)=\left(\mathbb{R}^{2} / \Gamma, h\right)$ as in Example 4 are locally reducible, of type Example 1 (iv). In fact, in local product coordinates in the universal cover $\widetilde{M}=\widetilde{N} \times S^{2}=\mathbb{R}^{2} \times S^{2}$ involving $\mathbb{R}^{2}$-coordinates $s$, $t$ as above, we have the product decomposition

$$
\tilde{g}=d t^{2}+\left[d s^{2}+\tau^{-2}(s) h_{c}\right]
$$

for the pull-back $\widetilde{g}$ of $g$ to $\widetilde{M}$.

On the other hand, if a compact oriented surface $(N, h)$ satisfying the hypotheses stated in Example 4 gives, by that construction, a fourmanifold of type Example 1 (iv), then $N$ is a torus and $h$ is obtained as above from a non-constant positive periodic solution $\tau$ to (23). To prove this, set $\tau=K+c>0$. By Lemma 4 (i), the metric $h_{0}=\tau^{-2} h$ is flat, so that $N$ is diffeomorphic to $T^{2}$ and (18) holds.

From (18) and (21) it follows that

$$
3 h_{0}(d \tau, d \tau)=\tau^{2}\left(3 c \tau^{2}-2 \tau^{3}-\lambda^{3}\right)
$$

and $\Delta_{0} \tau$ is a function of $\tau, \Delta_{0}=\tau^{2} \Delta$ being the Laplacian of $h_{0}$. Viewing $\tau$ and the flat metric $h_{0}$ as pulled back to the universal cover $\mathbb{R}^{2}$ of $N$, we can apply Lemma 7 to $\tau$ and $\left(\mathbb{R}^{2}, h_{0}\right)$, obtaining a global Killing field $X$ on $\left(\mathbb{R}^{2}, h_{0}\right)$, orthogonal to $d \tau$. Since $d \tau$ is invariant under a lattice group $\Gamma$, it follows from the uniqueness assertion of LEMMA 7 that $X$ is $\Gamma$-invariant up to multiplication by constants, which in turn easily implies that $X$ is a constant vector field on $\mathbb{R}^{2}$. Thus, we can choose coordinates $s, t$ on $\mathbb{R}^{2}$ such that $h_{0}=d s^{2}+d t^{2}$ and the flow of $X$ consists of $t$-translations. Now $\tau=\tau(s)$ and (24) gives (23). Note that $c>\lambda>0$ in view of Lemma 6 and LEMMA 8.

TOME $116-1988-\mathrm{N}^{\circ} 2$ 


\section{Existence of compact surfaces with the properties required in Example 4}

In this section we shall establish the existence of metrics $h$ satisfying the conditions stated in Example 4 on all compact orientable surfaces $N$ of genus $p \neq 1$. For $p=1$, we have already found such metrics on $T^{2}$ (Example 5); however, those metrics are geometrically less interesting, since the four-manifolds obtained from them as in Example 4 are locally reducible. Our existence proofs are based on the following result.

THEOREM (The Bifurcation Theorem : the case of simple multiplicity; [3, p. 155]). - Suppose that E, $E^{\prime}$ are real Banach spaces, $U$ is a neighborhood of zero in $E, I$ an interval of $\mathbb{R}$ and $f: U \times I \rightarrow E^{\prime} a$ $C^{2}$ map with the notation $f=f(x, \lambda)$ and $f_{x}, f_{\lambda}, f_{\lambda x}$ for the "partial" derivatives of $f$. Assume that $f(0, \lambda)=0$ for all $\lambda \in I$ and, for some $\lambda_{0} \in I, A=f_{x}\left(0, \lambda_{0}\right)$ is a Fredholm operator $E \rightarrow E^{\prime}$ of index 0 with $\operatorname{dim} \operatorname{Ker} A=1$, while for any non-zero $z \in \operatorname{Ker} A, f_{\lambda x}\left(0, \lambda_{0}\right) z$ is not in the image of $A$.

Then, near $\left(0, \lambda_{0}\right)$ there is exactly one (up to changes of parameter) continuous curve

$$
(-1,1) \ni t \longmapsto(x(t), \lambda(t)) \in U \times I
$$

such that $f(x(t), \lambda(t))=0$ for all $t, x(0)=0, \lambda(0)=\lambda_{0}$ and $x(t) \neq 0$ for $t \neq 0$.

Let $\left(N, h_{0}\right)$ be a compact surface of constant curvature $K_{0}$, orientable or not. For a function $x$ on $N$ and $\lambda$ in the interval $I=\left(\max \left(0, K_{0}\right), \infty\right)$ let us define $P(x, \lambda)$ to be the function $(K+c)^{3}-3(K+c) \Delta K-6|d K|^{2}-\lambda^{3}$, where $c=\lambda-K_{0}$ and the symbols $K, \Delta,||$ correspond to the metric $h=e^{2 x} h_{0}$ on $N_{0}$. Thus

$$
\begin{aligned}
P(x, \lambda)=[ & \left.e^{-2 x}\left(K_{0}+\Delta_{0} x\right)+\lambda-K_{0}\right]^{3} \\
& -3 e^{-2 x}\left[e^{-2 x}\left(K_{0}+\Delta_{0} x\right)+\lambda-K_{0}\right] \Delta_{0}\left[e^{-2 x}\left(K_{0}+\Delta_{0} x\right)\right] \\
& -6 e^{-2 x}\left|d\left[e^{-2 x}\left(K_{0}+\Delta_{0} x\right)\right]\right|_{0}^{2}-\lambda^{3}
\end{aligned}
$$

where $\Delta_{0}=-h_{0}^{i j} \nabla_{i} \nabla_{j}$ and ||$_{0}$ correspond to the metric $h_{0}$. Fix an integer $k \geq 7$. The Sobolev space $L_{k}^{2}=L_{k}^{2}(N)$ of functions with $k$ derivatives in $L^{2}$ can be made into a commutative Banach algebra with the pointwise multiplication, and so the self-mapping $x \mapsto e^{x}$ of $L_{k}^{2}$ is $C^{\infty}$. Therefore, (25) defines a $C^{\infty}$ mapping $P: L_{k+4}^{2} \times I \rightarrow L_{k}^{2}$. For any $\lambda \in I$,

$$
\begin{aligned}
P(0, \lambda) & =0, \\
P_{x}(0, \lambda) & =-3 \lambda\left(\Delta_{0}-\lambda\right)\left(\Delta_{0}-2 K_{0}\right), \\
P_{\lambda x}(0, \lambda) & =-3\left(\Delta_{0}-2 \lambda\right)\left(\Delta_{0}-2 K_{0}\right),
\end{aligned}
$$


where the subscripts are used for partial derivatives.

Remark 5. - We shall apply the Bifurcation Theorem to find solutions $(x, \lambda) \in L_{k+4}^{2} \times I$ to $P(x, \lambda)=0$, other than those with $x=0$. Note that for any such solution sufficiently close to the curve $\lambda \mapsto(0, \lambda), x$ is necessarily an analytic function on $N$ (in a manner compatible with either local holomorphic structure determined by $h_{0}$ ). In fact, as $k \geq 7$, $L_{k+4}^{2} \subset C^{9}(N)$. Thus, $h=e^{2 x} h_{0}$ is a $C^{9}$ metric on $N$ with (21). Moreover, it satisfies (20) since it is $C^{2}$ close to a metric of constant curvature $K_{0}$ with $K_{0}+c=\lambda>0$. Hence $h$ is analytic in suitable local coordinates (Remark 2). These coordinates coincide with those making $h_{0}$ analytic, since the transitions between them are conformal maps between analytic metrics on open sets in $\mathbb{R}^{2}$. Consequently, $x$ is analytic.

We shall say that two Riemannian metrics are homothetic (resp., locally homothetic) if one of them is isometric to a constant multiple of the other (resp., if this holds after restricting both metrics to suitable non-empty open subsets of the underlying manifolds).

Remark 6. - For a non-flat metric $h$ with Gauss curvature $K$ on a compact surface $N$, the number $\zeta(h)=\min |K| / \max |K|$ is a homothety invariant of $h$. If $h$ is analytic, $\zeta(h)$ is also a local homothety invariant (since a local homothety in $N$ then gives a global one on the universal cover of $N)$. Thus, if $t \mapsto h(t)$ is a continuous curve of analytic metrics on a compact surface $N$ of genus $p \neq 1$, which contains metrics with non-constant curvature as well as ones of constant curvature, then the metrics $h(t)$ represent uncountably many distinct local homothety types, i.e., they contain an uncountable family of metrics, no two of which are locally homothetic. In fact, the continuous function $t \mapsto \zeta(h(t))$ is nonconstant, so it assumes uncountably many values.

Proposition 2. - Let $h_{0}$ be a metric of constant curvature 1 on $\mathbb{R} P^{2}$ with a fixed effective action of $S^{1}$ by isometries. For any positive integer $m$ there exists a continuous curve $t \mapsto x(t, m)$ of $S^{1}$-invariant analytic functions on $\mathbb{R} P^{2}$ and a continuous curve $t \mapsto \lambda(t, m) \in(1, \infty)$, both parametrized by $t$ near 0 in $\mathbb{R}$, such that

(i) The $S^{1}$-invariant metric $h=h(t, m)=e^{2 x(t, m)} h_{0}$ on $\mathbb{R} P^{2}$ satisfies (20) and (21) with $\lambda=\lambda(t, m), c=c(t, m)=\lambda(t, m)-1$. For $t \neq 0$, $h(t, m)$ has non-constant curvature.

(ii) $h(0, m)=h_{0}$, i.e. $x(0, m)=0$ and $\lambda(0, m)=2 m(2 m+1)$, the $m$-th eigenvalue of the Laplacian of $\left(\mathbb{R} P^{2}, h_{0}\right)$. Thus, the local homothety 
invariant $c(t, m) / \lambda(t, m)$ of $h(t, m), t \neq 0$ (cf. Remark 4) satisfies :

$$
\lim _{t \rightarrow 0} \frac{c(t, m)}{\lambda(t, m)}=1-\frac{1}{2 m(2 m+1)} .
$$

(iii) For $m \neq m^{\prime}$ and any $t, t^{\prime} \neq 0, h(t, m)$ and $h\left(t^{\prime}, m^{\prime}\right)$ are not locally homothetic. For any fixed $m$, the metrics $h(t, m)$ represent uncountably many distinct local homothety types.

Proof. - Let $E$ (resp. $E^{\prime}$ ) be the Banach space of all $S^{1}$-invariant functions in $L_{k+4}^{2}$ (resp., in $L_{k}^{2}$ ), where $k \geq 7$ is fixed and let

$$
f: E \times(1, \infty) \longrightarrow E^{\prime}
$$

be the restriction of the map $P$ given by (25). The $m$-th eigenvalue of the Laplacian $\Delta_{0}$ of $\left(\mathbb{R} P^{2}, h_{0}\right)$ is $2 m(2 m+1)$ and its eigenspace consists of all $\mathbb{R}^{3}$-harmonic homogeneous polynomials of degree $2 m$ on $\mathbb{R}^{3}$, pushed down onto $\mathbb{R} P^{2}$ via restriction to the unit sphere $\left[2\right.$, p. 166]. Hence $2=2 K_{0}$ is not an eigenvalue and so, by $(26), f$ will satisfy the hypotheses of the bifurcation theorem at any $\lambda_{0}$ with $\operatorname{dim} \operatorname{Ker} f_{x}\left(0, \lambda_{0}\right)=1$.

We claim that this is the case for $\lambda_{0}=2 m(2 m+1)$ with any integer $m \geq 1$, i.e., that the subspace of $S^{1}$-invariant elements in each eigenspace of $\Delta_{0}$ is one-dimensional. To see that, choose Cartesian coordinates $t$, $y, z$ in $\mathbb{R}^{3}$ such that our $S^{1}$ action on $\mathbb{R} P^{2}$ comes from the rotations around the $t$-axis. The elements of $\operatorname{Ker} f_{x}\left(0, \lambda_{0}\right)$ with $\lambda_{0}=2 m(2 m+1)$ come from polynomials invariant under such rotations, which one easily sees by averaging. These invariant polynomials clearly have the form $Q\left(t^{2}, y^{2}+z^{2}\right)$, where $Q=Q(\alpha, \beta)$ is a homogeneous $m$-th order polynomial in two variables, while $\mathbb{R}^{3}$-harmonicity means that

$$
2 \alpha \frac{\partial^{2} Q}{\partial \alpha^{2}}+2 \beta \frac{\partial^{2} Q}{\partial \beta^{2}}+\frac{\partial Q}{\partial \alpha}+2 \frac{\partial Q}{\partial \beta}=0
$$

It is easy to verify that the space of polynomials $Q$ with the properties just stated is one-dimensional. Therefore, we can apply the bifurcation theorem, obtaining curves $x(t, m) \in L_{k+4}^{2}$ and $\lambda(t, m) \in(1, \infty)$ with

$$
P(x(t, m), \lambda(t, m))=0
$$

and $x(0, m)=0, \lambda(0, m)=2 m(2 m+1)$, while $x(t, m) \neq 0$ unless $t=0$. For $t$ close to $0, x(t, m)$ is analytic (Remark 4$)$. The curvature of the metric $h=e^{2 x(t, m)} h_{0}, t \neq 0$, is.non-constant. In fact, if it was constant, we would have

$$
\Delta_{0}\left(e^{-x(t, m)}+a\right)=2\left(e^{-x(t, m)}+a\right)
$$


for some real $a\left[13\right.$, proof of THEOREM $\left.\mathrm{II}^{\prime}\right]$ and, since 2 is not an eigenvalue of $\Delta_{0}, x(t, m)$ would be constant, which contradicts $x(t, m) \neq 0$ in view of (27) and (25). Together with the definition of $P$ this proves (i) (note that (20) holds for $t$ close to 0 ), while (ii) is obvious. In view of (ii), for $m \neq m^{\prime}$ and $t, t^{\prime}$ close to 0 , local homothety invariants distinguish $h(t, m)$ from $h\left(t^{\prime}, m^{\prime}\right)$ unless $t=t^{\prime}=0$. Restricting our curves to shorter intervals if necessary and using Remark 6, we obtain (iii), which completes the proof.

Proposition 3 (R. Schoen, S. Wolpert, S. T. Yau [15, p. 279]). For any compact orientable surface $N$ of genus $p>1$, let $\Sigma(N)$ be the moduli space of metrics with constant curvature -1 on $N$ for which the first positive eigenvalue of the Laplacian has multiplicity one.

Then $\Sigma(N)$ is non-empty. Moreover, it is an open subset of the Teichmüller space of $N$ (as it is defined by an open condition), so that $\operatorname{dim} \Sigma(N)=6 p-6$.

Proposition 4. - Let $N$ be a compact orientable surface of genus $p>1$.

(i) Given a metric $h_{0}$ of constant curvature -1 on $N$ for which the first eigenvalue $\lambda_{1}\left(h_{0}\right)$ of the Laplacian has multiplicity one, there is a continuous curve $t \mapsto x\left(t, h_{0}\right)$ of analytic functions on $N$ and a continuous curve $t \rightarrow \lambda\left(t, h_{0}\right) \in(0, \infty)$, both defined for $t$ near 0 in $\mathbb{R}$, such that $x\left(0, h_{0}\right)=0, \lambda\left(0, h_{0}\right)=\lambda_{1}\left(h_{0}\right)$ and, for each $t \neq 0$, the metric $h=h\left(t, h_{0}\right)=e^{2 x\left(t, h_{0}\right)} h_{0}$ has non-constant curvature and satisfies (20) and (21) with $\lambda=\lambda\left(t, h_{0}\right), c=c\left(t, h_{0}\right)=\lambda\left(t, h_{0}\right)+1$.

The local homothety invariants $c\left(t, h_{0}\right) / \lambda\left(t, h_{0}\right)$ of $h\left(t, h_{0}\right), t \neq 0$ (see Remark 3) satisfy

$$
\lim _{t \rightarrow 0} \frac{c\left(t, h_{0}\right)}{\lambda\left(t, h_{0}\right)}=1+\frac{1}{\lambda_{1}\left(h_{0}\right)} .
$$

(ii) The set of all $h\left(t, h_{0}\right)$ where $h_{0}$ satisfying the hypotheses of (i) may vary, contains a $(6 p-5)$-dimensional family of mutually non-homothetic metrics $h$ on $N$ having the properties required in Example 4 . For a fixed $h_{0}$, the metrics $h\left(t, h_{0}\right)$ represent uncountably many distinct local homothety types.

Proof. - In view of (26) the bifurcation theorem can be applied to $f=P: L_{k+4}^{2} \times(0, \infty) \rightarrow L_{k}^{2}$ defined by $(25)$ with $k \geq 7, h_{0}$ as in (i) and $\lambda_{0}=\lambda_{1}\left(h_{0}\right)$, giving solutions $(x(t), \lambda(t))=\left(x\left(t, h_{0}\right), \lambda\left(t, h_{0}\right)\right)$ to $P\left(x\left(t, h_{0}\right), \lambda\left(t, h_{0}\right)\right)=0$. Thus, for $t \neq 0, x\left(t, h_{0}\right)$ is non-constant and, clearly, $h\left(t, h_{0}\right)$ has non-constant curvature. For $t$ close to $0, x\left(t, h_{0}\right)$ is analytic (Remark 4) and $h=h\left(t, h_{0}\right)$ satisfies (20), which together with the definition of $P$ gives (i). On the other hand, two non-isometric metrics of curvature -1 on $N$ are not conformally equivalent, which implies (ii)

TOME $116-1988-\mathrm{N}^{\mathrm{o}} 2$ 
in view of Remark 6 and Proposition 3. This completes the proof.

Remark 7. - The bifurcation theorem can also be applied to finding metrics with (21) on compact surfaces of genus $1, e . g$. , by setting

$$
f(x, c)=\Delta_{0}\left[(K+c)^{3}-3(K+c) \Delta K-6|d K|^{2}\right]
$$

for $S^{1}$-invariant functions $x$ with $h_{0}$-mean value zero on a flat Klein bottle $\left(N, h_{0}\right)$ with Laplacian $\Delta_{0}$, where $K, \|$ and $\Delta$ correspond to the metric $h=e^{2 x} h_{0}$. However, the solutions $(x, c)$ to $f(x, c)=0$ with $x \neq 0$, obtained in this way near the curve $(0, c)$ of trivial solutions yield nothing else than the metrics of Example 5, the latter being projectable onto $N$ due to obvious $\mathbb{Z}_{2}$ symmetries of their defining functions $\tau$.

\section{New examples of compact four-manifolds with harmonic curvature}

In $[6$, p. 21] J.-P. Bourguignon posed the question of the existence of compact, simply connected Riemannian manifolds with harmonic curvature and with non-parallel Ricci tensor. The following result provides examples of such manifolds in dimension four.

THEOREM 4. - For any positive integer $m$ there is a continuous curve $t \mapsto g(t, m)$ of metrics with harmonic curvature on $S^{2} \times S^{2}$, parametrized by $t$ close to 0 in $\mathbb{R}$ and such that

(i) For $t \neq 0, g(t, m)$ is neither Einstein, nor conformally flat, nor locally reducible and satisfies (7) for either orientation.

(ii) $g(0, m)$ is the product metric $h_{0} \times\left(4 m^{2}+2 m-1\right)^{-1} h_{0}, h_{0}$ being a metric of constant curvature 1 on $S^{2}$.

(iii) If $m \neq m^{\prime}$, the metrics $g(t, m)$ and $g\left(t^{\prime}, m^{\prime}\right)$ are not locally homothetic for any $t, t^{\prime}$. For a fixed $m$, the metrics $g(t, m)$ represent uncountably many distinct local homothety types.

(iv) The norm $|W|$ of the Weyl tensor of $g(t, m)$ is positive everywhere and $\left(S^{2} \times S^{2},|W|^{2 / 3} \cdot g(t, m)\right)$ is the Riemannian product of two surfaces diffeomorphic to $S^{2}$, one of which has constant curvature.

(v) For $t \neq 0$, the isometry group of $g(t, m)$ is $\mathbb{Z}_{2} \times \mathrm{O}(2) \times \mathrm{O}(3)$ with $\mathrm{O}(3)$ acting on the second factor of $S^{2} \times S^{2}$ and $\mathbb{Z}_{2} \times \mathrm{O}(2)$ on the first, $\mathbb{Z}_{2}$ being generated by the antipodal mapping.

Proof. - Applying the construction of Example 4 to $N=S^{2}$ and to the pull-back $h$ of the metric $h(t, m)$ on $\mathbb{R} P^{2}$ described in Proposition 2, we obtain metrics $g(t, m)$ with harmonic curvature on $S^{2} \times S^{2}$. Now (i) and (iv) are immediate from Example 4 and the discussion in Example 5, and (ii) from Proposition 2. Assertion (v) follows from the fact that 
$g(t, m)$ and the product metric $h \times h_{c}$ with $c=c(t, m)$, conformal to $g(t, m)$, have the same isometries, as they determine each other, even locally (Example 4). This also implies that $h$ is determined by the local geometry of $g(t, m)$, so that (iii) is immediate from Proposition 2 (iii), which completes the proof.

Using TheOREM $4(\mathrm{v})$ and the fact that $\mathbb{Z}_{2} \times \mathrm{O}(2) \subset \mathrm{O}(3)$ contains conjugates to all elements of order 2 , we easily obtain :

Corollary 2. - Consider the obvious action of $\mathrm{O}(3) \times \mathrm{O}(3)$ on $S^{2} \times S^{2}$ and let $\Gamma$ be a finite subgroup acting freely, so that $\Gamma$ is isomorphic to $\mathbb{Z}_{2} \times \mathbb{Z}_{2}$, or to $\mathbb{Z}_{2}$, or trivial. Then $\Gamma$ is conjugate to a subgroup of the isometry group of each metric $g(t, m)$ described in THEOREM 4 . In particular, $\left(S^{2} \times S^{2}\right) / \Gamma$ admits a family of metrics sharing all the local properties of the $g(t, m)$.

The principal $\mathrm{SO}(3)$ bundles over a compact surface $N$ are classified by their second Stiefel-Whitney class [19, p. 798], so that there are only two such bundles. They both admit flat connections unless $N$ is the 2-sphere. In fact, such a connection (and the bundle) is determined by the conjugacy class of its holonomy homomorphism

$$
\sigma: \pi_{1} N \longrightarrow \mathrm{SO}(3)
$$

For $\sigma$ which cannot be lifted to $\operatorname{Spin}(3)$ we obtain a flat connection in a bundle that does not admit a spin structure, i.e., in the non-trivial principal $\mathrm{SO}(3)$ bundle over $N$.

Using Example 4, Proposition 4 and the discussion in Example 5, and applying the same argument as in the proof of Tнеовем 4, we obtain

THEOREM 5. - Let $N$ be a compact orientable surface of genus $p>1$.

(i) Either $\mathrm{SO}(3)$ bundle $M$ with fibre $S^{2}$ over $N$ admits a family of metrics $g=g\left(t, h_{0}, \sigma\right)$ with harmonic curvature, which are neither Einstein, nor conformally flat, nor locally reducible, unless $t=0$, and satisfy (7) for either local orientation.

They are parametrized by a metric $h_{0}$ of constant curvature -1 on $N$ having the first positive eigenvalue of the Laplacian with multiplicity one, a homomorphism

$$
\sigma: \pi_{1} N \longrightarrow \mathrm{SO}(3)
$$

and a real number $t$ close to 0 , and $(M, g)$ is obtained as in Example 4 from the metric $h=h\left(t, h_{0}\right)$ on $N$ described in Proposition 4 and from $\sigma$.

(ii) The bundle $M \rightarrow N$ is trivial if and only if $\sigma$ can be lifted to $\operatorname{Spin}(3)$.

$$
\text { TOME } 116-1988-\mathrm{N}^{\circ} 2
$$


(iii) The homothety type of $g\left(t, h_{0}, \sigma\right)$ determines the isometry type of $h_{0}$ and the conjugacy class of $\sigma$. For fixed $h_{0}$ and $\sigma$ the metrics $g\left(t, h_{0}, \sigma\right)$ give rise to uncountably many distinct local homothety types on $M$.

(iv) The norm $|W|$ of the Weyl tensor of $g=g\left(t, h_{0}, \sigma\right)$ is positive everywhere and $|W|^{2 / 3} g$ is a locally reducible metric admitting the $S^{2}$ fibres of $M$ as factors and inducing on them metrics of constant curvature.

Letting $\mathrm{SO}(3)$ act on $\mathbb{R} P^{2}$ instead of $S^{2}$, we obtain

Corollary 3. - The assertion of ThEOREM 5 remains true if $S^{2}$ is replaced everywhere by $\mathbb{R} P^{2}$.

Remark 8. - The action of $\pi_{1} N$ on $\widetilde{N} \times S^{2}$ in Example 4 is holomorphic. Thus, the metric $g$ defined there is Hermitian with respect to a suitable complex structure on $M$ and it is globally conformal to the Kähler metric $|W|^{2 / 3} g$. This is true in particular for the metrics with harmonic curvature obtained in Theorems 4 and 5.

\section{BIBLIOGRAPHIE}

[1] Atryah (M. F.), Hitchin (N. J.) and Singer (I. M.). - Self-duality in fourdimensional Riemannian geometry, Proc. Roy. Soc. London Ser. A, t. 362, 1978, p. $425-461$.

[2] Berger (M.), Gauduchon (P.) and Mazet (E.). - Le Spectre d'une Variété Riemannienne. - Berlin-Heidelberg-New York, Springer-Verlag, (Lecture Notes in Math., 194), 1971.

[3] Berger (M. S.). - Nonlinearity and Functional Analysis. - New York-San Francisco-London, Academic Press, 1977.

[4] Besse (A. L.). - Einstein Manifolds, Berlin-Heidelberg, Springer-Verlag, (Ergebnisse der Mathematik und ihrer Grenzgebiete, 10), 1987.

[5] Bourguignon (J.-P.). - Les variétés de dimension 4 à signature non nulle dont la courbure est harmonique sont d'Einstein, Invent. Math., t. 63, 1981, p. 263-286.

[6] Bourguignon (J.-P.). - Metrics with harmonic curvature, Global Riemannian Geometry, [T. J. WillmoRe and N. Hitchin, eds.], p. 18-26. - Chichester, Ellis Horwood Ltd., 1984.

[7] CAlABi (E.). - Extremal Kähler metrics, Seminar on Differential Geometry, [S. T. YAU, ed.], p. 259-290. - Princeton, N.J., Princeton University Press, 1982.

[8] DERDZIŃSKI (A.). - Classification of certain compact Riemannian manifolds with harmonic curvature and non-parallel Ricci tensor, Math. Z., t. 172, 1980, p. 273-280.

[9] DerdzińSKI (A.). - Self-dual Kähler manifolds and Einstein manifolds of dimension four, Compositio Math., t. 49, 1983, p. 405-433.

[10] Derdziński (A.) and Shen (C.-L.). - Codazzi tensor fields, curvature and Pontryagin forms, Proc. London Math. Soc. (3), t. 47, 1983, p. 15-26.

[11] DeTurck (D.) and Goldschmidt (H.). - Preprint, Philadelphia, Univ. of Pennsylvania, 1984 . 
[12] GRAY (A.). - Invariants of curvature operators of four-dimensional Riemannian manifolds, Proc. 13th Biennial Seminar Canadian Math. Congress, vol. 2, p. 42$65,1972$.

[13] OвAтA (M.). - The conjectures on conformal transformations of Riemannian manifolds, Bull. Amer. Math. Soc., t. 77, 1971, p. 265-270.

[14] Polom во (A.). - Nombres caractéristiques d'une surface kählérienne compacte, C. R. Acad. Sci. Paris Sér. A Math., t. 283, 1976, p. 1025-1028.

[15] Schoen (R.), Wolpert (S.) and Yau (S. T.). - Geometric bounds on the low eigenvalues of a compact surface, Proc. Sympos. Pure Math., vol. 36, p. 279-285, 1980 .

[16] Schouten (J. A.). - Ricci Calculus. - Berlin-Heidelberg, Springer-Verlag, 1954 .

[17] Singer (I. M.) and Thorpe (J. A.). - The curvature of 4-dimensional Einstein spaces, Global Analysis, [Papers in Honor of K. KodAIrA; D. C. SPENCER and S. IYANAGA, eds.], p. 355-365. - Princeton, N.J., Princeton University Press, 1969 .

[18] ThORPe (J.). - Some remarks on the Gauss-Bonnet integral, J. Math. Mech., t. 18, 1969, p. $779-786$.

[19] Whitney (H.). - Topological properties of differentiable manifolds, Bull. Amer. Math. Soc., t. 43, 1937, p. 785-805. 